\title{
HALVING THE MILNOR MANIFOLDS AND SOME CONJECTURES OF RAY
}

\author{
DAVID M. SEGAL ${ }^{1}$
}

\begin{abstract}
The peculiar properties of the $2^{j}-2$ dimensional generators of unitary bordism (the 2-primary Milnor generators) are related to the $2^{j}-3$ dimensional indecomposable torsion classes of Alexander. This result is then used to confirm a conjecture of Ray concerning the generalised homology spectral sequence for $M S p_{*}(M U)$. Finally it is noted that Ray's conjecture to the effect that all classes in $M S p_{*}$ are detectable by $K O$-characteristic numbers must fail.
\end{abstract}

1. Halving the Milnor manifolds. We consider the cofibration sequence of spectra

$$
M S p \stackrel{j}{\longrightarrow} M U \stackrel{p}{\longrightarrow} M U / M S p ;
$$

$M U / M S p$ will be the Thom spectra for $(U, S p)$ relative bordism. Recall that $M U_{*}=Z\left[x_{1}, x_{2}, \cdots, x_{i}, \cdots\right], x_{i} \in M U_{2 i}$ and that for $i=2^{k}-1$ the $x_{i}$ (the 2-primary Milnor generators) have the peculiarity that, if $h_{*}$ is the Hurewicz map, then $h_{*}\left(x_{i}\right)$ is divisible by 2 in $H_{2 i}(M U ; Z)$ although of course $x_{i}$ is not divisible in $M U_{*}$. We will now shed some light on this peculiar behavior.

J. C. Alexander [1] has defined a series of indecomposable torsion elements $\mu_{i} \in M S p_{8 i-3}, 2 \mu_{i}=0$. Let $\sigma_{1}$ be the generator of $M S p$. In all that follows $n$ will be equal to $2^{k-2}$. Let $\partial: M U / M S p \rightarrow S M S p$ be the cofibre of $p$. Since $j_{*} \mu_{n}=0$, we can choose $\bar{\mu}_{n} \in(M U / M S p)_{8 n-2}$ such that $\partial_{*} \bar{\mu}_{n}=\mu_{n}$. Let

$$
f_{k}: S^{8 n-3} \rightarrow M S p ; \quad g_{k}: S^{8 n-2} \rightarrow M U / M S p
$$

classify $\mu_{n}, \bar{\mu}_{n}$ respectively; $\partial \circ g_{k} \cong S f_{k}$. Let $U_{2} \in H^{0}\left(M S p ; Z_{2}\right)$ be the Thom class and $s^{i} \in H^{i}\left(S^{i} ; Z_{2}\right)$ the generator. Then Alexander has shown that $f_{k}$ is detected by a functional cohomology operation, namely,

$$
S q_{f_{k}}^{2 \Delta(k)}\left(U_{2}\right)=s^{8 n-3}
$$

Received by the editors October 27, 1972.

AMS (MOS) subject classifications (1970). Primary 57D90, 55B20; Secondary 55H25.

Key words and phrases. Unitary bordism, symplectic bordism, Milnor manifolds, Hattori-Stong conjecture.

${ }^{1}$ This research was supported by the Research Foundation of the City University of New York. 
or, suspending once,

$$
S q_{S_{f k}}^{2 \Delta(k)}\left(S U_{2}\right)=s^{8 n-2}
$$

But $\partial^{*}\left(S U_{2}\right)=0$ so

$$
g_{k}^{*} S q_{\partial}^{2 \Delta(k)}\left(S U_{2}\right)=s^{8 n-2}
$$

and $g_{k}$ can be detected by cohomology in the ordinary way. $\left(S q^{2 \Delta(k)}\right.$ denotes $S q^{(0,0,0, \cdots, 0,2,0, \cdots)}(2$ in the $k$ th position) in the Milnor basis notation.) Recall the definition of functional operations and compute $S q_{\partial}^{2 \Delta(k)}\left(S U_{2}\right)$ :

$$
S\left[S q_{\partial}^{2 \Delta(k)}\left(S U_{2}\right)\right]=(S p)^{*} S q^{2 \Delta(k)}(S j)^{*-1} U_{2} .
$$

Now $(S j)^{*-1}\left(U_{2}\right)$ is well defined; it is just (the suspension of) the mod 2 Thom class of $M U$; call it $U_{2}^{\prime} . S q^{2 \Delta(k)} U_{2}^{\prime}$ is the primitive class in the coalgebra $H^{8 n-2}\left(M U ; Z_{2}\right)$. Thus taking normal Chern numbers on $(M U / M S p)_{*}$ we have that $s_{(4 n-1)}(c)\left[\bar{\mu}_{n}\right]$ is odd. Since $p_{*}(2 \bar{\mu})=2 \mu_{n}=0$ we can pull $2 \bar{\mu}_{n}$ back to $M U_{8 n-2}$ and the pull back can be chosen as $x_{4 n-1}$, a 2primary Milnor generator. In other words, the 2-primary Milnor generators (properly chosen) can be halved by being regarded as classes in the relative $(U, S p)$-bordism theory. Note that if we used $(U, f r)$ theory instead we could halve $x_{1}$ but not the higher 2-primary Milnor generators; see the discussion of $(U, f r)$ theory in [2].

2. A conjecture of Ray. We use the preceding observations to establish a conjecture due essentially to Ray [5] concerning the generalised homology (Atiyah-Hirzebruch) spectral sequence

$$
E_{* *}^{2}=H_{*}(M U ; M S p) \Rightarrow M S p_{*}(M U) .
$$

We identify $E_{0, i}^{2}$ with $M S p_{i}$ and $E_{i, 0}^{2}$ with $H_{i}(M U ; Z)$.

THEOREM 2.1. The class of $\mu_{n} \in E_{0,8 n-3}^{2}$ persists nonzero to $E_{0,8 n-3}^{8 n-2}$ where it is killed by a transgressive differential from a class in $H_{8 n-2}(M U ; Z)$ which is half of the Hurewicz image of a (properly chosen) $x_{4 n-1}$.

Proof. Since $M U_{i}(M S p)=0$ for $i$ odd it is clear that $\mu_{n}$ is killed.

Consider the commutative diagram (2.2):

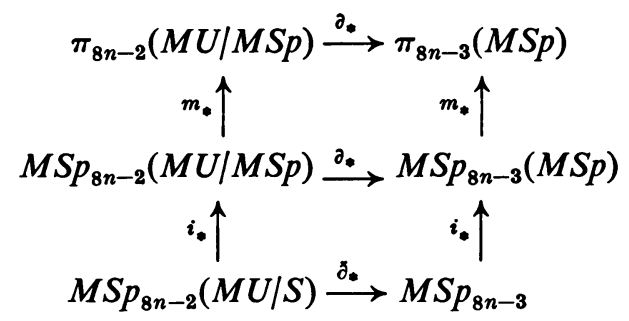


Explanation of (2.2) $M U / S$ is the Thom spectrum of $(U, f r)$ bordism, $i$ is the map of the cofibration sequence

$$
S \stackrel{\bar{j}}{\longrightarrow} M U \stackrel{\bar{p}}{\longrightarrow} M U / S
$$

to the cofibration sequence (1.1) and $\bar{\partial}: M U / S \rightarrow S^{1}$ is the cofibre of $\bar{p}$. The maps $m_{*}$ arise from the fact that (1.1) is a sequence of spectra with compatible $M S p$ actions and so the $(U, S p)$ relative bordism sequence is a long exact sequence of $M S p_{*}$ modules.

Since $\bar{j}_{*} \mu_{n}=0, \mu_{n}$ is in the image of $\bar{\partial}_{*}$. The assertion that $\left[\mu_{n}\right] \neq 0 \in$ $E_{0.8 n-3}^{8 n-2}$ is equivalent to the statement that $\mu_{n}$ is not in the image of the composition

$$
M S p_{8 n-2}\left([M U / S]^{(8 n-3)}\right) \longrightarrow M S p_{8 n-2}(M U / S) \stackrel{\delta_{\bullet}}{\longrightarrow} M S p_{8 n-3}
$$

(where $[X]^{(r)}$ denotes the $r$-skeleton of $X$ ).

Now suppose that

$$
y_{n} \in M S p_{8 n-2}(M U / S)
$$

is in the image of $M S p_{8 n-2}\left([M U / S]^{(8 n-3)}\right)$ and that $\bar{\partial}_{*} y_{n}=\mu_{n}$. Then $\partial_{*}\left[m_{*} i_{*} y_{n}\right]=m_{*} i_{*} \bar{\partial}_{*} y_{n}=m_{*} i_{*} \mu_{n}=\mu_{n}$; therefore $m_{*} i_{*} y_{n} \in(M U / M S p)_{8 n-2}$ is a $\bar{\mu}_{n}$, 'half of a Milnor generator' and can be represented by a map $g_{k}: S^{8 n-2} \rightarrow M U / M S p$ which we know to be detected in $Z_{2}$-cohomology by (the anti-image under $p^{*}$ of) the primitive Chern class; in other words, the image of the map

$$
g_{k^{*}}: H_{8 n-2}\left(S^{8 n-2} ; Z\right) \rightarrow H_{8 n-2}(M U / M S p ; Z)
$$

contains an odd integral multiple of the image under $p_{*}$ of an indecomposable element of $H_{8 n-2}(M U ; Z)$. But this is inconsistent with the supposition that $g_{k^{*}}$ can be factored through $H_{8 n-2}\left(M S p \wedge M U / S^{(8 n-3)}\right)$. (Observe that $m_{*}$ is, in homology, determined by the ordinary product on $H_{*}(M U)$.) So $\left[\mu_{n}\right] \neq 0 \in E_{0,8 n-3}^{8 n-2}$. Finally, the image of

$$
y_{n} \in H_{8 n-2}(M U ; Z)
$$

will be $j_{*}^{-1} h_{*} m_{*} i_{*} y_{n}=j_{*}^{-1} h_{*} \bar{\mu}_{n}$. We choose $x_{4 n-1}$ so that $j_{*} x_{4 n-1}=2 \bar{\mu}_{n}$ and we are finished.

3. Ray's Hattori-Stong conjecture. In [6] we left open the question of whether $M S p_{31}$ is 0 or $Z_{2}$. Here we note that in fact since in the Adams' spectral sequence for $M S p$ the map $k_{1}: E_{3}^{2.28}(M S p) \rightarrow E_{3}^{3.34}(M S p)$ given by multiplication by $k_{1}$ is an isomorphism onto, and since $k_{1}$ and all elements of $E_{3}^{2.28}(M S p)$ are permanent cycles it follows that all elements of $E_{3}^{3.34}(M S p)$ are permanent cycles; $d_{3}\left(v_{4}^{2}\right) \neq 0$ by the results of [3] or [7] and so $M S p_{31}=$ $Z_{2}$. This gives us a class in $M S p_{*}$ which can not be detected by $K O$ characteristic numbers and so Ray's ' $\bmod 2 M S p$ Hattori-Stong conjecture' [4] is false. 
Note. S. Kochman has informed me that in certain details the spectral sequence calculations in [6] are in error, e.g. $d_{2} v_{4}=\gamma_{0,2}+\gamma_{0.1} v_{2}$ not $\gamma_{0.2}$ as claimed. These errors do not affect the validity of the above observations. Kochman also finds the Hattori-Stong conjecture to be false but on other grounds.

We hope to discuss the question of the nonvanishing of three-fold products of torsion elements further in a subsequent note.

4. Acknowledgements. We wish to thank J. C. Alexander, N. Ray and $\mathrm{S}$. Kochman for informing us of their various studies of $M S p_{*}$. We also wish to point out that Alexander has obtained the results of $\S 1$ by other methods; his results will appear in Topology.

\section{BiBLIOGRAPHY}

1. J. C. Alexander, A family of indecomposable symplectic manifolds, Amer. J. Math. 94 (1972), 699-710.

2. P. E. Conner and E. E. Floyd, The relation of cobordism to K-theories, Lecture Notes in Math., no. 28, Springer-Verlag, Berlin and New York, 1966. MR 35 \#7344.

3. E. E. Floyd, Stiefel-Whitney numbers of quaternionic and related manifolds, Trans. Amer. Math. Soc. 155 (1971), 77-94. MR 42 \#8509.

4. N. Ray, A note on the symplectic bordism ring, Bull. London Math. Soc. 3 (1971), 159-162. 282.

5. —_ The symplectic bordism ring, Proc. Cambridge Philos. Soc. 71 (1972), 271-

6. D. M. Segal, On the symplectic cobordism ring, Comment. Math. Helv. 45 (1970), 159-169. MR 41 \#7699.

7. - Divisibility conditions on characteristic numbers of stably symplectic manifolds, Proc. Amer. Math. Soc. 27 (1971), 411-415. MR 42 \#5282.

Department of Mathematics, City College of the City University of New York, NEW YORK, NEW YORK 10031 